\title{
Gamma-ray emission from Cassiopeia A produced by accelerated cosmic rays
}

\author{
E. G. Berezhko ${ }^{1}$, G. Pühlhofer ${ }^{2}$, and H. J. Völk ${ }^{2}$ \\ ${ }^{1}$ Institute of Cosmophysical Research and Aeronomy, 31 Lenin Ave., 677891 Yakutsk, Russia \\ e-mail: berezhko@ikfia.ysn.ru \\ 2 Max Planck Institut für Kernphysik, Postfach 103980, 69029 Heidelberg, Germany \\ e-mail: Gerd.Puehlhofer@mpi-hd.mpg.de
}

Received 23 September 2002 / Accepted 7 January 2003

\begin{abstract}
The nonlinear kinetic model of cosmic ray (CR) acceleration in supernova remnants (SNRs) is used to describe the relevant properties of Cassiopeia A (Cas A). In order to reproduce the SNR's observed size, expansion rate and thermal X-ray emission we employ a piecewise homogeneous model for the progenitor's circumstellar medium developed by Borkowski et al. (1996). It consists of a tenuous inner wind bubble, a dense shell of swept-up red supergiant wind material, and a subsequent red supergiant wind region. A quite large SNR interior magnetic field $B_{\mathrm{d}} \approx 1 \mathrm{mG}$ is required to give a good fit for the radio and X-ray synchrotron emission. The steep radio spectrum is consistent with efficient proton acceleration which produces a significant shock modification and leads to a steep electron spectrum at energies $\epsilon_{\mathrm{e}}<1 \mathrm{GeV}$. The calculated integral $\gamma$-ray flux from Cas A, $F_{\gamma} \propto \epsilon_{\gamma}^{-1}$, is dominated by $\pi^{0}$-decay $\gamma$-rays due to relativistic protons. It extends up to roughly $30 \mathrm{TeV}$ if $\mathrm{CR}$ diffusion is as strong as the Bohm limit. At TeV energies it satisfactorily agrees with the value $5.8 \times 10^{-13} \mathrm{~cm}^{-2} \mathrm{~s}^{-1}$ detected by the HEGRA collaboration.
\end{abstract}

Key words. supernovae: individual: Cassiopeia A - cosmic rays - gamma rays: theory - acceleration of particles shock waves - radiation mechanisms: non-thermal

\section{Introduction}

Cassiopeia A (Cas A) is a prominent shell type supernova remnant (SNR), and a bright source of synchrotron radiation observed at radio frequencies (e.g. Bell et al. 1975; Tuffs 1986; Braun 1987; Andersen et al. 1991; Kassim et al. 1995) and most probably also in the X-ray band (Allen et al. 1997; Favata et al. 1997). Since SNRs are widely suspected to be the main sources of the Galactic cosmic rays (CRs) up to energies of at least $10^{15} \mathrm{eV}$, this youngest Galactic SNR offers an important possibility to test this CR origin hypothesis. With the synchrotron interpretation, the measured emission of hard X-rays is direct evidence for the existence of a large number of relativistic electrons with energies up to about $10 \mathrm{TeV}$, presumably accelerated at the outer SNR shock. Experimental information about nuclear CR production can only be obtained by high energy $\gamma$-ray measurements. If protons are accelerated in Cas A to at least the same energy and as efficiently as electrons then the $\pi^{0}$-decay $\gamma$-ray spectrum, created in their hadronic collisions with the background nuclei, should extend to energies above $1 \mathrm{TeV}$ with a hard power-law. The detection of a signal in $\mathrm{TeV} \gamma$-rays has been indeed recently reported by the HEGRA

Send offprint requests to: H. J. Völk,

e-mail: Heinrich.Voelk@mpi-hd.mpg.de collaboration (Aharonian et al. 2001). Therefore it is of the exceptional importance to find out whether this $\gamma$-ray emission is consistent with a hadronic origin.

In this paper a thorough theoretical analysis will be given, based on earlier work (Berezhko et al. 2001). It follows similar analyses for SN 1006 (Berezhko et al. 2002) and for Tycho's SNR (Völk et al. 2002). We note that in the meantime also new $\gamma$-ray observations of SNR RX J1713.7-3946 by the CANGAROO experiment have been discussed (Enomoto et al. 2002) and criticized (Reimer \& Pohl 2002; Butt et al. 2002) on a phenomenological basis. Unfortunately, the latter object is up to now only very poorly understood, for example regarding the type of explosion (quasi-explosive nuclear burning of an accreting White Dwarf vs. core collapse of a massive progenitor star) and the distance (estimates range from $1 \mathrm{kpc}$ to $6 \mathrm{kpc}$ ). The object also lies in an environment of complex morphology. In addition the radio observations are so scarce that they do not allow to determine a spectrum. As a result only phenomenological single box-type estimates for the particle spectra which are responsible for the synchrotron/Inverse Compton radiation and the hadronic $\pi^{0}$-decay $\gamma$-ray emission have been made up to now, preventing any firm conclusion regarding the nature of the $\gamma$-ray emission. Even a detailed theoretical model like ours could, with the currently available data, only lead to a number 
of constraints. In contrast, Cas A is perhaps the best-studied object of its kind. The following analysis makes detailed use of this impressive multi-wavelength knowledge.

The chemical compositions of characteristic parts of the supernova (SN) ejecta (the fast moving knots, e.g. Reed et al. 1995 and references therein) and of the circumstellar material (the quasi-stationary flocculi, Peimbert 1971; Peimbert \& van den Bergh 1971; Kirshner \& Chevalier 1977; Chevalier \& Kirshner 1978) suggest that the progenitor star started as a massive object. It should have evolved from a hot main sequence star with a tenuous fast wind to a red supergiant phase with a slow but dense wind, and finally into a hot Wolf-Rayet star with a fast wind again, sweeping up part of the preceeding red supergiant material into a dense shell before exploding as a core collapse SN (Chevalier \& Liang 1989; Garcia-Segura et al. 1996). In this spirit Borkowski et al. (1996) modeled the thermal X-ray emission as well as the size and the expansion rate of the present SNR from their result that the outer blast wave had already passed the swept-up red supergiant wind shell and was presently propagating through the unperturbed slow red supergiant wind. According to the X-ray measurements of Favata et al. (1997) the SNR shock has swept up about $5 M_{\odot}$ of this circumstellar material. Therefore we can expect a detectable level of $\pi^{0}$-decay $\gamma$-ray flux.

Empirical arguments for such a structure of the circumstellar material around the presupernova star come for example from the radio observations of Cas A. Despite its youth of about 320 years, characterized also by the relatively small amount of matter swept up by the SN shock, the observed radio flux undergoes considerable secular decline (e.g. Rees 1990), which amounts to about $0.6 \%$ per year. For a uniform circumstellar material one would expect the radio flux to increase until an intermediate Sedov stage of evolution, corresponding to an age of about $10^{3} \mathrm{yr}$. Only in the case when the SN shock propagates through a wind of the progenitor star with progressively decreasing density $\rho \propto r^{-2}$, the total radio emission is expected to decrease with time (e.g. Berezhko \& Ksenofontov 1999).

This suggests two rather complementary approaches regarding the production of nonthermal particles and their radiation. One can either emphasize the strong radio emission from small inhomogeneities whose free energy (kinetic and magnetic) is assumed to lead to stochastic acceleration of energetic electrons which are responsible for the synchrotron emission from the radio to the X-ray band (Scott \& Chevalier 1975; Dickel \& Greisen 1979; Cowsik \& Sarkar 1984; Jones et al. 1994; Atoyan et al. 2000b). This gives also rise to an attendant nonthermal Bremsstrahlung (NB) and Inverse Compton (IC) component extending to the high energy $\gamma$-rays. In this phenomenological scenario the large scale SNR blast wave does not have to play an important role for electron energization, but it may still be the main source for the nonthermal nuclear particle component (Atoyan et al. 2000a). An inventory of the radio to high energy $\gamma$-ray emission along these lines has been made phenomenologically by Atoyan et al. (2000b,a), including detailed fits to the observed synchrotron spectrum.

Alternatively one can to lowest order ignore the role of small-scale inhomogeneities for the production of the very high energy nonthermal particle component. At least as its carriers they are in difficulty on the argument that high energy particles (ultrarelativistic electrons and nuclear particles) require a large acceleration/propagation volume given by spatial scales $L \sim \lambda_{\mathrm{mfp}}(p) \times c / u$. Here $u$ denotes a characteristic nonrelativistic speed of mass motions, $c$ is the speed of light, and $\lambda_{\operatorname{mfp}}(p)$ is the scattering mean free path that increases with momentum $p$. For strong scattering $\lambda_{\mathrm{mfp}}=\mathrm{O}\left(r_{\mathrm{g}}\right)$, where $r_{\mathrm{g}} \propto p$ is the particle gyro radius. At least for the highest energy particles $L$ amounts to a few percent of the remnant radius. Ignoring the dynamical effects underlying the small-scale emission features, the dominant remaining large-scale entropy generator is the SNR blast wave. It has then to be investigated which of the observed nonthermal features can be explained by shock accelerated particles.

In this paper we shall apply the nonlinear kinetic model for diffusive shock acceleration in SNRs (Berezhko et al. 1996; Berezhko \& Völk 1997, 2000a,b) to the specific case of Cas A. This spherically symmetric model should describe the global properties of the nonthermal emission especially at high energies, even though small-scale processes may indeed introduce some modifications at low particle energies, contributing especially to the radio flux. The calculation of the nonthermal particle populations is a central part of the theory which also determines the spherically symmetric portion of the overall gas dynamics in the remnant selfconsistently.

In fact, we shall attempt to investigate whether the global properties of Cas A are consistent with the idea that the SN blast wave is the main source of energetic particles and in particular, whether the observed $\gamma$-ray emission is consistent with a hadronic origin.

\section{Model}

To describe the circumstellar medium we use the specific model of Borkowski et al. (1996). Accordingly, part of the slow red supergiant wind of the SN progenitor has been swept up into a dense shell by a fast stellar wind during the final blue supergiant (probably Wolf-Rayet) phase of the progenitor star. Therefore the inner circumstellar medium consists of three zones: a tenuous wind-blown bubble, a dense shell, and a freely expanding red supergiant wind. The outer circumstellar regions that were generated during the main sequence phase play no role here.

We describe the profile of gas number density $N_{\mathrm{g}}=\rho / m_{\mathrm{p}}$ in the analytic form

$N_{\mathrm{g}}=\frac{N_{\mathrm{bsh}}+N_{\mathrm{w}}}{2}+\frac{N_{\mathrm{w}}-N_{\mathrm{bsh}}}{2} \tanh \left(\frac{r-R_{2}}{l}\right)$,

where

$N_{\mathrm{bsh}}=\frac{N_{\mathrm{b}}+N_{\mathrm{sh}}}{2}+\frac{N_{\mathrm{sh}}-N_{\mathrm{b}}}{2} \tanh \left(\frac{r-R_{1}}{l}\right)$,

and

$N_{\mathrm{w}}=N_{\mathrm{w} 2}\left(R_{2} / r\right)^{2}$

is the gas number density of the free red giant wind (region $r>R_{2}$ ), $N_{\mathrm{b}}$ and $N_{\mathrm{sh}}$ correspond to the hot bubble (region $r<$ $R_{1}$ ) and shell (region $R_{1}<r<R_{2}$ ) respectively, $\rho$ is the gas 
density, $m_{\mathrm{p}}$ is the proton mass. This interpolation formula provides a smooth transition between the above three zones on the scale $l$ which is taken small enough, $l \ll R_{1}$, that its concrete value does not influence the final results.

We use the same type of formula (1) and (2) for the magnetic field profile $B_{0}(r)$ with $B_{\mathrm{b}}, B_{\text {sh }}$ and

$B_{\mathrm{w}}=B_{\mathrm{w} 2} R_{2} / r$

for the bubble, shell and wind regions respectively.

The SN explosion ejects an expanding amount of matter with energy $E_{\mathrm{sn}}$ and mass $M_{\mathrm{ej}}$. During an initial period the ejecta have a wide distribution in radial velocity $v$. The fastest part of these ejecta is described by a power law $\mathrm{d} M_{\mathrm{ej}} / \mathrm{d} v \propto v^{2-k}$ (Jones et al. 1981). The interaction of the ejecta with the circumstellar medium creates a strong shock which will diffusively accelerate particles.

The acceleration model consists in a selfconsistent solution of the CR transport equation together with the gas dynamic equations in spherical symmetry (Berezhko et al. 1996; Berezhko \& Völk 1997), in an extension of this model to the case of a nonuniform circumstellar medium (Berezhko \& Völk 2000b).

The CR diffusion coefficient is taken at the Bohm limit

$\kappa(p)=\kappa(m c)(p / m c)$,

where $\kappa(m c)=m c^{3} /(3 e B) ; e, m$ and $p$ are the particle charge, mass and momentum, respectively; $B$ is the magnetic field strength; and $c$ is the speed of light.

In the downstream region the magnetic field is assumed to be frozen into the gas and is described by the equation

$\frac{\partial \boldsymbol{B}}{\partial t}=\nabla \times(\boldsymbol{w} \times \boldsymbol{B})$,

where $\boldsymbol{w}$ is the gas mass speed, directed everywhere radially in our spherical model. The boundary condition for this equation is the relation between the postshock magnetic field $B_{2}$ and the upstream field at the shock position $B_{\mathrm{S}}=B_{0}\left(R_{\mathrm{S}}\right)$. The tangential field component $B_{\perp}$ is amplified at the shock front because of compression, so that its postshock value is $B_{2 \perp}=\sigma B_{0 \perp}$, where $\sigma$ is the shock compression ratio. Therefore we have $B_{2}=\sigma_{B} B_{0}$, where $\sigma_{B}=\sqrt{\left(\sigma^{2}-1\right) \sin ^{2} \phi+1}, \phi$ being the angle between the upstream magnetic field $\boldsymbol{B}_{0}\left(R_{\mathrm{S}}\right)$ and the shock normal. For simplicity we suggest that the downstream magnetic field is purely tangential and use $\sigma_{B}=\sigma$.

The number of suprathermal protons injected into the acceleration process is described by a dimensionless injection parameter $\eta$ which is a fixed fraction of the ISM particles entering the shock front. For simplicity it is assumed that the injected particles have a velocity four times higher than the postshock sound speed. Unfortunately there is no complete selfconsistent theory of a collisionless shock transition, which can predict the value of the injection rate and its dependence on the shock parameters. For the case of a purely parallel shock hybrid simulations predict quite a high ion injection (e.g. Scholer et al. 1992; Bennet \& Ellison 1995) which corresponds to the value $\eta \sim 10^{-2}$ of our injection parameter. Such a high injection is consistent with analytical theory (Malkov \& Völk 1995, 1996;
Malkov 1998) and confirmed by measurements near the Earth's bow shock (Trattner \& Scholer 1994). We note however that in our spherically symmetric model these results can only be used with some important modification. The circumstellar magnetic field may be assumed to be strongly perturbed at least in the shell region. Efficient particle injection takes place only on those portions of the shock surface which are currently locally quasiparallel. On other parts of the shock where it is more and more oblique, the magnetic field essentially suppresses the leakage of suprathermal particles from the downstream region back upstream (Ellison et al. 1995; Malkov \& Völk 1995). Therefore the mean injection rate and subsequent $\mathrm{CR}$ production efficiency, properly averaged over the entire shock surface, is expected to be considerably lower compared with the case of a purely parallel shock. Due to this fact the number of accelerated CRs, calculated within our spherically symmetric model, has to be corrected (decreased) by some renormalisation factor $f_{\text {re }}<1$, because of the lack of efficient injection/acceleration on the part $1-f_{\text {re }}$ of the actual shock surface.

We assume that electrons are also injected into the diffusive shock acceleration process still at nonrelativistic energies below $m_{\mathrm{e}} c^{2}$. Since the electron injection mechanism is not very well known (e.g. Malkov \& Drury 2001), for simplicity we consider their acceleration starting from the same momentum as protons. At relativistic proton energies they have exactly the same dynamics as the protons. Therefore, neglecting synchrotron losses, their distribution function at any given time has the form

$f_{\mathrm{e}}(p)=K_{\mathrm{ep}} f(p)$

for relativistic proton energies, with some constant factor $K_{\text {ep }}$ which is about $10^{-2}$ for the average CRs in the Galaxy. We take it here as a parameter and use relation (7) only at the injection momentum in order to determine the number of injected/accelerated electrons for a given proton injection rate. At all other momenta the electron distribution function is found by solving the transport equation.

In fact the calculated electron distribution function $f_{\mathrm{e}}(p)$ deviates from expression (7) only at sufficiently large momenta because of synchrotron losses, which are taken into account by supplementing the ordinary diffusive transport equation with a loss term:

$$
\frac{\partial f_{\mathrm{e}}}{\partial t}=\nabla \kappa \nabla f_{\mathrm{e}}-\boldsymbol{w} \nabla f_{\mathrm{e}}+\frac{\nabla \boldsymbol{w}}{3} p \frac{\partial f_{\mathrm{e}}}{\partial p}+\frac{1}{p^{2}} \frac{\partial}{\partial p}\left(\frac{p^{3}}{\tau_{1}} f_{\mathrm{e}}\right),
$$

where the first three terms on the right hand side of this equation describe diffusion, convection due to the mass velocity $\boldsymbol{w}$ of the gas, and adiabatic effects, respectively. The synchrotron loss time in the fourth term is determined by the expression (e.g. Berezinskii et al. 1990)

$\tau_{1}=\left(\frac{4 r_{0}^{2} B^{2} p}{9 m_{\mathrm{e}}^{2} c^{2}}\right)^{-1}$,

where $m_{\mathrm{e}}$ is the electron mass and $r_{0}$ the classical electron radius.

The solution of the dynamic equations at each instant of time yields the CR spectrum and the spatial distributions of 
CRs and gas. This allows us to calculate the expected flux $F_{\gamma}^{\pi}\left(\epsilon_{\gamma}\right)$ of $\gamma$-rays from $\pi^{0}$-decay because of hadronic (p-p) collisions of CRs with the gas nuclei. Following the work of Dermer (1986) and its later improvement by Naito \& Takahara (1994) we use here the isobar model at the proton kinetic energies $\epsilon_{\mathrm{k}}<3 \mathrm{GeV}$ and the scaling model at $\epsilon_{\mathrm{k}}>7 \mathrm{GeV}$ with a linear connection between 3 and $7 \mathrm{GeV}$. This model agrees very well with the simpler approach, introduced by Drury et al. (1994) (see also Berezhko \& Völk 1997, 2000a; Berezhko et al. 1999) at high energies $\epsilon_{\gamma}>0.1 \mathrm{GeV}$, except in the cutoff region, where the scaling model yields a significantly smoother turnover of the $\gamma$-ray spectrum at somewhat lower energies.

The choice of $K_{\text {ep }}$ allows us to determine in addition the electron distribution function and to calculate the associated emission (for details, see Berezhko et al. 2002, in a recent analysis of SN 1006). We calculate here the IC radiation taking into account as target photon fields - besides the cosmic microwave background - the infrared field with a mean photon energy of $\epsilon_{\mathrm{ph}}=0.01 \mathrm{eV}$ and an energy density of $0.2 \mathrm{eV} / \mathrm{cm}^{3}$, and the optical field with a mean photon energy of $\epsilon_{\mathrm{ph}}=1.5 \mathrm{eV}$ and an energy density of $0.5 \mathrm{eV} / \mathrm{cm}^{3}$ (e.g. Drury et al. 1994; Gaisser et al. 1998).

\section{Results and discussion}

\subsection{Supernova dynamics}

In the current approach, we have reduced the circumstellar density profile everywhere by a factor of 1.8 compared with what was derived by Borkowski et al. (1996). This corresponds to the parameter values:

$$
\begin{aligned}
& N_{\mathrm{b}}=0.17 \mathrm{~cm}^{-3}, \quad N_{\mathrm{sh}}=11 \mathrm{~cm}^{-3}, \quad N_{\mathrm{w} 2}=2 \mathrm{~cm}^{-3}, \\
& R_{1}=3.8 \times 10^{18} \mathrm{~cm}, \quad R_{2}=4.94 \times 10^{18} \mathrm{~cm} .
\end{aligned}
$$

Together with the renormalization factor $f_{\text {re }}$ which is discussed later, the lower circumstellar density is the main reason for the significant reduction of the expected $\gamma$-ray flux (see below) compared to our previous calculations which did not take into account $f_{\text {re }}$ and were based on a swept-up mass value of $8 M_{\odot}$ (Berezhko et al. 2001). The density reduction is close to that suggested by the X-ray analysis of the BeppoSAX data (Favata et al. 1997), which yielded $5 M_{\odot}$ instead of $8 M_{\odot}$ (Vink et al. 1996; Borkowski et al. 1996) for the swept-up mass.

In order to reproduce the observed shock size $R_{\mathrm{S}}$ and its expansion rate $V_{\mathrm{S}}$ the following SN parameters are used: explosion energy $E_{\mathrm{sn}}=4 \times 10^{50} \mathrm{erg}$, ejecta mass $M_{\mathrm{ej}}=2 M_{\odot}$, and power-law index $k=6$ for the ejecta velocity distribution (cf. Berezhko et al. 2002). As distance to Cas A we adopt $d=3.4 \mathrm{kpc}$ (cf. Reed et al. 1995).

The results of our calculations together with the experimental data are shown in Fig. 1-4. In Fig. 1a we also show the profiles of the gas number density $N_{\mathrm{g}}\left(R_{\mathrm{S}}\right)$ and magnetic field $B_{0}\left(R_{\mathrm{S}}\right)$, upstream of the shock front that is at position $R_{\mathrm{S}}(t)$.

As can be seen in Fig. 1a, after the shock has entered the shell region its speed drops during the initial 60 years by more than a factor of ten and then remains almost constant up to the current epoch.

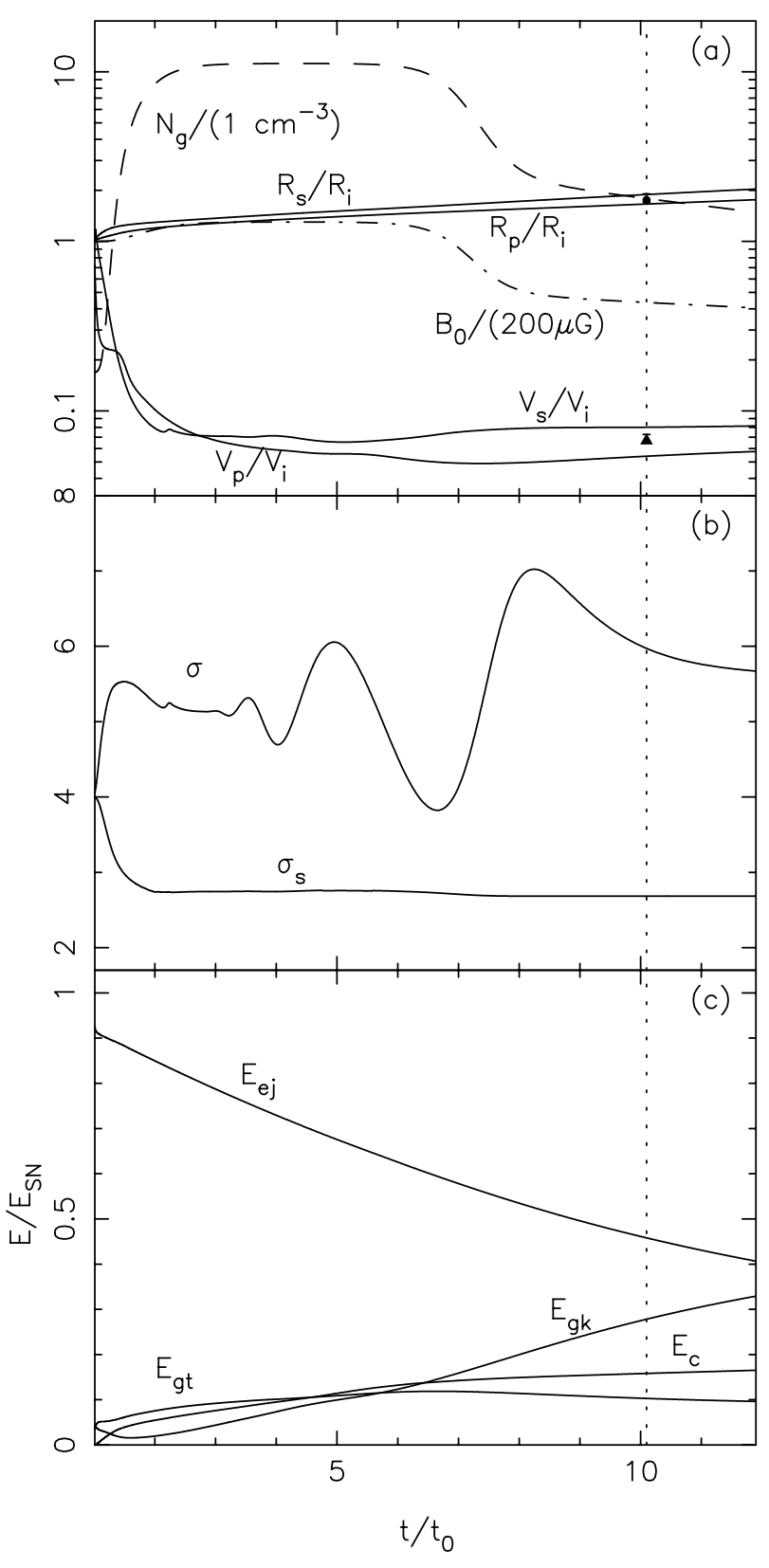

Fig. 1. a) Shock (contact discontinuity) radius $R_{\mathrm{S}}\left(R_{\mathrm{p}}\right)$ and shock (contact discontinuity) speed $V_{\mathrm{s}}\left(V_{\mathrm{p}}\right)$; b) total shock $(\sigma)$ and subshock $\left(\sigma_{\mathrm{s}}\right)$ compression ratios; c) ejecta $\left(E_{\mathrm{ej}}\right), \mathrm{CR}\left(E_{\mathrm{c}}\right)$, gas thermal $\left(E_{\mathrm{gt}}\right)$ and gas kinetic $\left(E_{\mathrm{gk}}\right)$ energies as a function of time. The vertical dotted line corresponds to the current evolutionary stage. The circumstellar gas number density $N_{\mathrm{g}}\left(R_{\mathrm{S}}\right)$ and magnetic field $B_{0}\left(R_{\mathrm{S}}\right)$ profiles are shown in a) by dashed and dash-dotted lines, respectively. Scale values are $R_{\mathrm{i}}=1 \mathrm{pc}, V_{\mathrm{i}}=30000 \mathrm{~km} \mathrm{~s}^{-1}, t_{0}=31.7$ years. The observed size (Reed et al. 1995) and speed (Andersen \& Rudnick 1995) of the shock are shown by the full circle and triangle, respectively, in a).

At the current epoch $t \approx 320 \mathrm{yr}$ the calculations reasonably reproduce the observed size (Reed et al. 1995) and expansion rate (Andersen \& Rudnick 1995) of the remnant.

A proton injection rate $\eta=2.5 \times 10^{-3}$ is used in order to provide the nonlinear shock modification required to reproduce quite a steep radio emission spectrum (see below). According to Fig. $1 \mathrm{~b}$ the shock is indeed strongly modified by the CR 


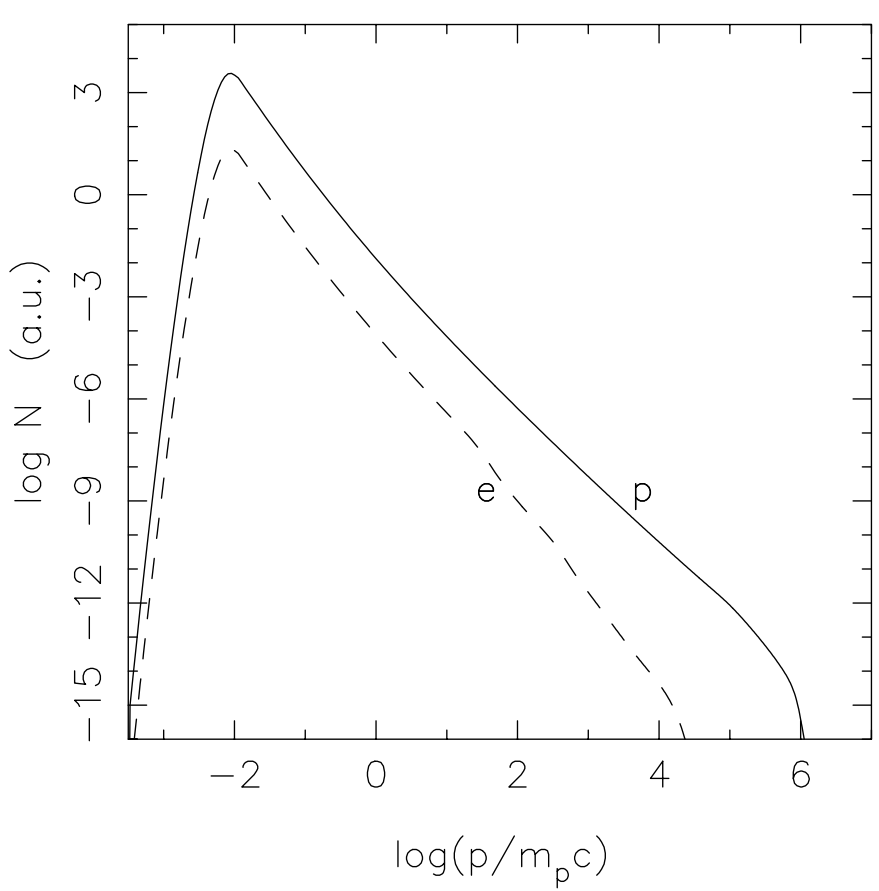

Fig. 2. The overall CR proton (solid line) and electron (dashed line) spectra as function of momentum.

backreaction: the total shock compression ratio $\sigma \approx 5.5$ exceeds the classical value 4 , whereas the subshock compression ratio is considerably smaller, $\sigma_{\mathrm{s}} \approx 2.7$.

\subsection{The accelerated $C R$ spectrum}

According to Fig. 1c about $15 \%$ of the explosion energy has been transformed into CRs at the current stage, that is $E_{\mathrm{c}} \approx$ $6 \times 10^{49}$ erg.

The overall momentum spectrum of accelerated protons

$N(p, t)=16 \pi^{2} p^{2} \int_{0}^{\infty} \mathrm{d} r r^{2} f(r, p, t)$,

presented in Fig. 2, turns over at about $p=10^{5} m_{\mathrm{p}} c$ and extends almost up to $p_{\max }=10^{6} m_{\mathrm{p}} c$ because of the extremely high magnetic field strength used: $B_{\mathrm{sh}}=200 \mu \mathrm{G}, B_{\mathrm{w} 2}=100 \mu \mathrm{G}$. The value $p_{\max }$ is limited by geometrical factors which are the finite size and speed of the shock, its deceleration and the adiabatic cooling effect in the downstream region (Berezhko 1996). If we approximate the overall proton spectrum by a power-law $N \propto p^{-\gamma}$ then the power-law index slowly decreases from $\gamma \approx 3$ at $p \lesssim m_{\mathrm{p}} c$ to $\gamma \approx 1.9$ at the highest momenta $10^{2} m_{\mathrm{p}} \lesssim p \lesssim 10^{5} m_{\mathrm{p}} c$ due to the strong shock modification.

The shape of the overall electron spectrum deviates from proportionality to the proton spectrum $N_{\mathrm{e}}(p) \propto N(p)$ at high momenta $p>p_{1} \approx 30 m_{\mathrm{p}} c$, because of the synchrotron losses in the downstream region where the magnetic field $B_{\mathrm{d}} \approx B_{2}$ is of the order of $1 \mathrm{mG}$. According to expression (9) the synchrotron losses become important for electron momenta greater than

$\frac{p_{1}}{m_{\mathrm{p}} c} \approx 1.3\left(\frac{10^{8} \mathrm{yr}}{t}\right)\left(\frac{10 \mu \mathrm{G}}{B_{\mathrm{d}}}\right)^{2}$.
Substituting the SN age $t=320 \mathrm{yr}$ into this expression we have $p_{1} \approx 30 m_{\mathrm{p}} c$, in good agreement with the numerical results (Fig. 2).

The shock constantly produces the electron spectrum $f_{\mathrm{e}} \propto$ $p^{-q}$ with $q \approx 4$ up to the maximum momentum $p_{\max }^{\mathrm{e}}$, which is much larger than $p_{1}$. Therefore, within the momentum range $p_{1}$ to $p_{\max }^{\mathrm{e}}$ due to the synchrotron losses, the electron spectrum is $f_{\mathrm{e}} \propto p^{-5}$ and the corresponding overall electron spectrum is $N_{\mathrm{e}} \propto p^{-3}$.

The maximum electron momentum can be estimated by equating the synchrotron loss time (9) and the acceleration time

$\tau_{\mathrm{a}}=\frac{3}{\Delta u}\left(\frac{\kappa_{1}}{u_{1}}+\frac{\kappa_{2}}{u_{2}}\right)$,

where $u_{1}=V_{\mathrm{s}}$ and $u_{2}=u_{1} / \sigma$ are the upstream and downstream gas velocities with respect to the shock front, and $\Delta u=u_{1}-u_{2}$. In the general case the downstream magnetic field equals $B_{2}=$ $\sigma_{\mathrm{B}} B_{0}$ and correspondingly

$\frac{p_{\max }^{e}}{m_{\mathrm{p}} c}=6.7 \times 10^{4}\left(\frac{V_{\mathrm{s}}}{10^{3} \mathrm{~km} \mathrm{~s}^{-1}}\right) \sqrt{\frac{(\sigma-1)}{\sigma\left(1+\sigma_{\mathrm{B}} \sigma\right)}\left(\frac{10 \mu \mathrm{G}}{B_{0}}\right)}$.

The main part of the electrons with the highest energies $\epsilon \gtrsim$ $10 \mathrm{TeV}$ is produced during shock propagation through the shell. At this stage $V_{\mathrm{s}} \approx 2500 \mathrm{~km} \mathrm{~s}^{-1}$ which leads to a maximum electron momentum $p_{\max }^{\mathrm{e}} \approx 10^{4} m_{\mathrm{p}} c$ (for simplicity we use $\sigma_{\mathrm{B}}=\sigma$ below), in agreement with the numerical results (Fig. 2).

According to the results from the next section, the energy content of the electrons at the current epoch is $E_{\mathrm{c}}^{\mathrm{e}}=K_{\mathrm{ep}} E_{\mathrm{c}}=$ $2.4 \times 10^{47} \mathrm{erg}$.

\subsection{The synchrotron emission from Cas $A$}

The parameter $K_{\mathrm{ep}}=4 \times 10^{-3}$ gives reasonable agreement between the calculated and the measured synchrotron emission in the radio- and X-ray ranges, as one can see from Fig. 3. The calculated synchrotron spectrum at the epoch 1970 (solid curve), which corresponds to that of the radio measurements of Baars et al. (1977), is compared with the experimental data. Also a calculated curve for the present epoch (dashed line), corresponding to the X-ray measurements, and a prediction for the epoch 2022 (dashed-dotted line) are given. The strong shock modification leads to a steep spectrum $N_{\mathrm{e}} \propto p^{-2.6}$ of sub-GeV accelerated particles (Fig. 2). As result of our choice of a large magnetic field $\left(B_{\mathrm{sh}}=200 \mu \mathrm{G}, B_{\mathrm{w} 2}=100 \mu \mathrm{G}\right)$ the calculation fits the radio data $S_{v} \propto v^{-\alpha}, \alpha \approx 0.8$, rather well. In fact, the electron spectrum has a concave shape at $p<p_{1} \approx 30 m_{\mathrm{p}} c$. This leads to a flattening of the synchrotron spectrum $S_{v}(v)$ at $v=10$ to $100 \mathrm{GHz}$, consistent with the experiment. Our calculated synchrotron flux fits even marginally the Mezger et al. (1986) measurements at $1.2 \mathrm{~mm}$ and the infrared emission measured at $6 \mu \mathrm{m}$ by Tuffs et al. (1997), even though, as one can see from Fig. 3 , these two points are above the pure power-law extrapolation $S_{v} \propto v^{-0.77}$. It is possible that the far infrared energy flux has a significant thermal component, cf. Braun (1987), Tuffs et al. (1997) and Vink (1999). The steepening of the electron spectrum at $p>p_{1}$ caused by synchrotron 


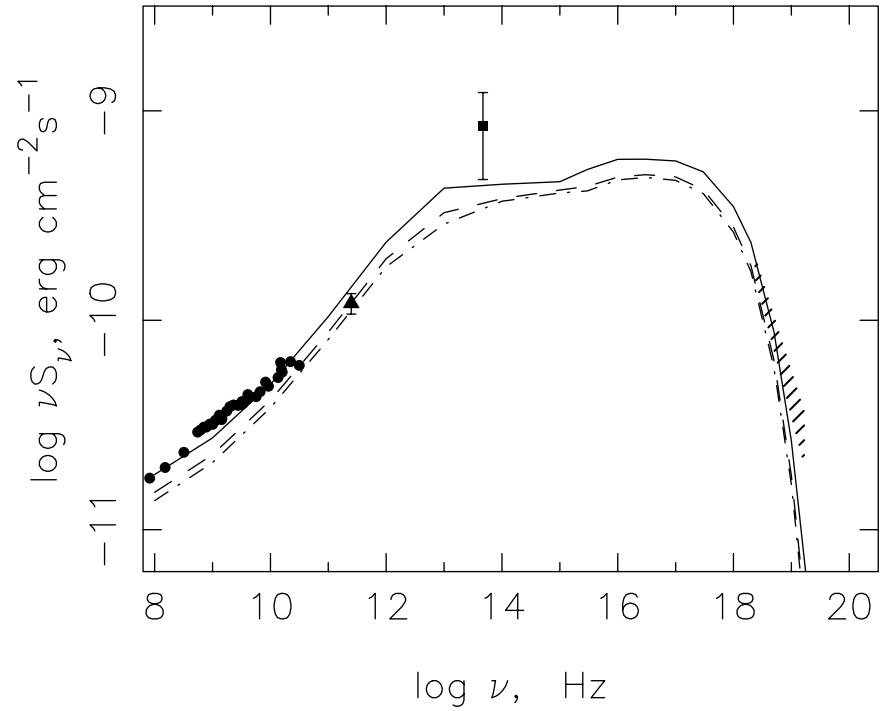

Fig. 3. Overall synchrotron spectral energy distributions as a function of frequency, calculated for the epochs 1970 (solid curve), 2002 (dashed curve), and 2022 (dashed-dotted curve). The radio-emission above $100 \mathrm{MHz}$ (Baars et al. 1977), the data at $1.2 \mathrm{~mm}$ (triangle) from Mezger et al. (1986) and $6 \mu \mathrm{m}$ (square) from Tuffs et al. (1997), as well as the hard X-ray spectrum (Allen et al. 1997) are presented. Due to the non-contemporaneous observations, the radio data should correspond to the solid curve, whereas the $\mathrm{X}$-ray data must be compared with the dashed curve. In the radio range theory and experiment agree fairly well. To achieve agreement also in the X-ray regime, a small increase of the overall electron cutoff momentum $p_{\max }$ of roughly $10 \%$ would be required (see text for details).

losses leads to a flat spectral energy distribution $v S_{v}$ that connects the nonthermal mid-infrared with the X-ray band (Fig. 3).

It is important to note that the complicated shape of the electron spectrum is naturally produced by the model. As far as the overall nonthermal spectrum is concerned, there is no strong need to add other sources of energetic particles. The bright "radio ring" is in any case explainable by the swept-up red supergiant wind shell which is contained in our model. It is another question whether individual "radio knots" are local acceleration regions or rather just magnetic field enhancements bathed in a pervasive particle background. For the nonthermal $\mathrm{X}$-ray and $\mathrm{TeV} \gamma$-ray emission they appear too small to locally accelerate the radiating multi-TeV particles in a stochastic manner. For a given frequency, a radio knot that consists of a local B-field enhancement will be illuminated by lower energy electrons than the ambient spatial regions. The strong nonlinear modification of the SN shock that generates such particles then ensures that these lower energy electrons have a steeper spectrum - just what distinguishes these knots form the average emission. Whether this effect is quantitatively sufficient, is a difficult question on the characteristics of the turbulent structure of the shocked red supergiant wind shell. However, it might go a long way to explain the $20 \%$ contribution (Tuffs 1986) of the radio knots to the total radio emission. Potential 2nd order Fermi acceleration in the turbulent shell, as proposed by Scott \& Chevalier (1975), cannot be ruled out. However, there is no indication for its action either. For diffusive shock acceleration at the blast wave, on the other hand, we know from theory that it can have the required high efficiency. And it can do it all. No other process needs to be invoked.

The shock modification can only be produced by the backreaction of the proton $\mathrm{CR}$ component. Therefore one can consider the extremely steep Cas A spectrum in the radio range as indirect evidence of efficient nuclear CR production. The other important physical factor is the high magnetic field value which leads to substantial synchrotron losses of electrons with energies $\epsilon_{\mathrm{e}}>30 \mathrm{GeV}$ : a magnetic field strength $B_{\mathrm{sh}}=200 \mu \mathrm{G}$ is needed to reproduce the observed radio and X-ray synchrotron fluxes. Since we assume that the postshock field $B_{2}=\sigma B_{\mathrm{s}}$, the downstream magnetic field amounts to about $B_{\mathrm{d}} \approx B_{2} \approx 1 \mathrm{mG}$ in the shell (Fig. 1a). This is roughly consistent with previous estimates (e.g. Atoyan et al. 2000b).

Such a large magnetic field $B_{0}(r)$ considerably exceeds the value for a red supergiant wind, assuming that its typical surface field at a radius of about $3 \times 10^{13} \mathrm{~cm}$ is about $1 \mathrm{G}$. We believe that the large field strength in the shell is likely either due to turbulent amplification of the red supergiant wind field by the shell formation in the final Wolf-Rayet phase or due to considerable field amplification near the shock by CR streaming (Lucek \& Bell 2000). If the second factor is relevant, the expected amplified field goes like $B_{0}\left(R_{\mathrm{S}}\right) \propto \sqrt{N_{\mathrm{g}}\left(R_{\mathrm{s}}\right)}$ (Bell \& Lucek 2001). Our field $B_{0}\left(R_{\mathrm{s}}\right)$ is distributed according to this relation. The deduced field amplification is still well within the upper bound set by the overall energy and momentum balance relations (Völk et al. 2002).

Our results show that even at the current epoch (when the SN shock propagates through the free red supergiant wind) the observed synchrotron emission is still determined by the electrons accelerated during the shock propagation through the shell. Therefore the magnetic field $B_{0}(r)$ in the wind zone is not a very relevant parameter for the fit to the observations.

According to Eq. (5), we note also that the exponential cutoff of the overall synchrotron spectrum at $v_{\max } \sim 10^{18} \mathrm{~Hz}$ is roughly independent of the magnetic field, as pointed out by Aharonian \& Atoyan (1999) for the case of a uniform field. Indeed the emission with frequency $v \sim v_{\max }$ is produced by electrons with momenta $p \sim p_{\max }^{\mathrm{e}}$ from their cutoff region. Since the maximum power of the synchrotron emission of electrons with momentum $p$ is emitted at frequency $v \propto \sigma_{B} B_{0} p^{2}$ (e.g. Berezinskii et al. 1990), and taking into account that $p_{\max }^{\mathrm{e}} \propto V_{\mathrm{s}} /\left(\sigma_{B} \sqrt{B_{0}}\right)$ we conclude that $v_{\max } \propto V_{\mathrm{s}}^{2} / \sigma_{B}$, independent of $B$. A roughly $20 \%$ increase in $v_{\max }$ would be required to bring the overall $\mathrm{X}$-ray data into close agreement with the contemporaneous model calculations (dashed curve), while leaving the low-frequency ranges unchanged at all epochs (the low-energy electron spectrum is independent of the cutoff energy). Whereas the spectral hardening beyond the radio range is a basic feature of nonlinear shock acceleration, this is not true for the precise value of the overall high-energy cutoff. This cutoff depends on the detailed characteristics of the scattering wave field at long wavelengths. With the above approximation of the diffusion coefficient by the Bohm limit, we see that a $20 \%$ increase in the characteristic value of $V_{\mathrm{s}}^{2} / \sigma_{B}$ is well within the variations of these quantities over the shell region 


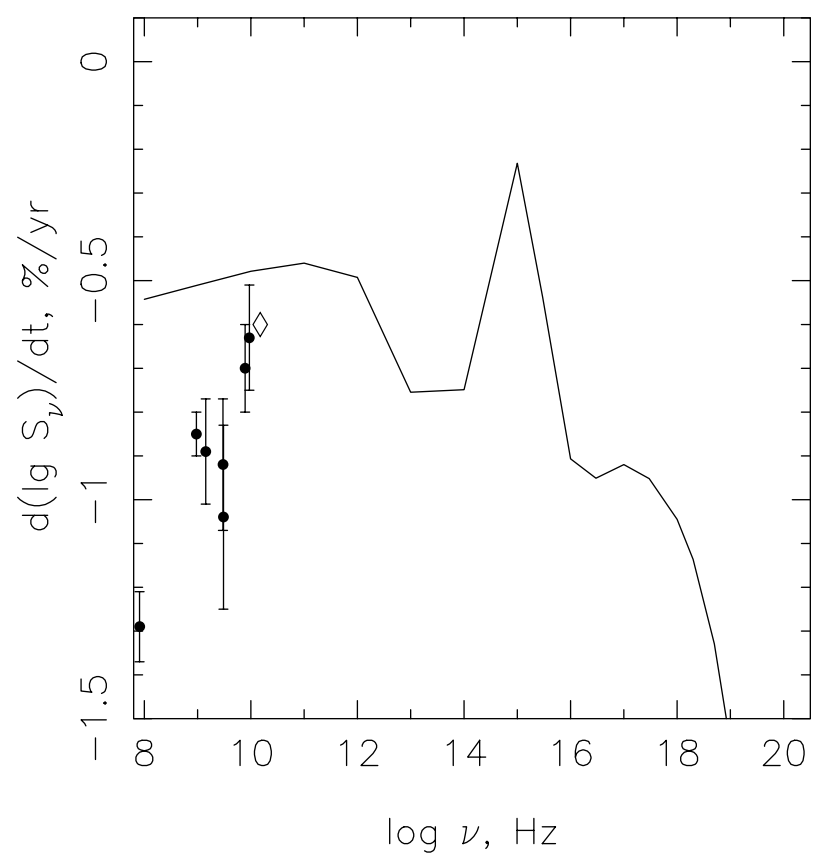

Fig. 4. The rate of secular decline of the total flux of synchrotron radiation. The diamond shows the recent result of O'Sullivan \& Green (1999) at $15 \mathrm{GHz}$. All other data points are from Rees (1990).

(Fig. 3). Thus we can consider the agreement between theoretical results and observations as remarkably good.

\subsection{The secular decline of the radio flux}

Important information about the circumstellar parameters comes from the measured secular decline of the radio flux which is presented in Fig. 4. In the case of a very young SNR the population of CRs would be expected to be an increasing function of time, if the remnant was expanding into a homogeneous medium with constant density and magnetic field. For Cas A, even the existence of the decline of the radio flux itself is a strong indication that the $\mathrm{SN}$ shock at the current evolutionary stage propagates through a region of monotonically decreasing magnetic field strength $B_{0}\left(R_{\mathrm{s}}\right)$ and density $\rho_{0}\left(R_{\mathrm{s}}\right)$. The most natural assumption, proposed by Borkowski et al. (1996), identifies this region with the wind region. If we were to suppose that the $\mathrm{CR}$ population and the radio emission from its electron component are dominated by particles accelerated in this free wind region, the synchrotron flux would scale as

$S_{v} \propto B_{0}\left(R_{\mathrm{s}}\right)^{(\gamma+1) / 2} N_{0}\left(R_{\mathrm{s}}\right)$,

where we represented the spectrum of radio emitting electrons in power-law form

$N_{\mathrm{e}}(p)=N_{0}\left(p / p_{0}\right)^{-\gamma}$.

In the wind region we have $B_{0}\left(R_{\mathrm{S}}\right) \propto R_{\mathrm{S}}^{-1}$, the number of accelerated electrons scales as the amount of swept-up mass $N_{0} \propto R_{\mathrm{s}}$, and therefore

$S_{v} \propto R_{\mathrm{s}}^{-(\gamma-1) / 2}$.
In our case $V_{\mathrm{s}} \approx 2400 \mathrm{~km} \mathrm{~s}^{-1}, R_{\mathrm{s}} \approx 2 \mathrm{pc}$ and $\gamma \approx 2.6$. For the secular flux decline

$\frac{\mathrm{d} \lg S_{v}}{\mathrm{~d} t}=-\frac{\gamma-1}{2} \frac{V_{\mathrm{s}}}{R_{\mathrm{s}}}$

we would therefore have $\mathrm{d} \lg S_{v} / \mathrm{d} t \approx-0.1 \% \mathrm{yr}^{-1}$. Its absolute value is considerably smaller than the observed one (Fig. 4).

The explanation for the large observed value of the secular decline of the radio flux comes from the idea that the radio emission is still dominated by the contribution of electrons accelerated at the previous stage during shock propagation through the dense shell. In this case the electrons undergo adiabatic cooling because of the expansion of the downstream region, and one can rewrite relation (14) in the form

$S_{v} \propto B_{\mathrm{d}}^{(\gamma+1) / 2} a^{-(\gamma+2)}$,

where $a=\left(\rho_{\mathrm{i}} / \rho\right)^{1 / 3}$ is the adiabatic factor which describes the particle adiabatic cooling, $\rho$ is the gas density in the region where electrons are confined, and $\rho_{\mathrm{i}}$ is its value at the epoch when these electrons were accelerated. It is natural to assume that the downstream magnetic field is frozen into the gas. In this case it changes according to the relation $B_{\mathrm{d}} \propto a^{-2}$. Let us assume that at the current epoch every downstream volume element has $a \propto R_{\mathrm{s}}$. Then the expected decline of the synchrotron flux is

$\frac{\mathrm{d} \lg S_{v}}{\mathrm{~d} t}=-(2 \gamma+3) \frac{V_{\mathrm{s}}}{R_{\mathrm{s}}}$,

which gives $\mathrm{d} \lg S_{v} / \mathrm{d} t \approx-0.8 \% \mathrm{yr}^{-1}$. This value is in the observed range $\mathrm{d} \lg S_{v} / \mathrm{d} t \approx-1.0 \% \mathrm{yr}^{-1}$ to $-0.6 \% \mathrm{yr}^{-1}$ (see Fig. 4). We take also into account that a revision of the data made by Rees (1990) led him to the conclusion that the secular decrease of the flux of Cas A is about $-0.8 \% \mathrm{yr}^{-1}$ and is independent of frequency. This is consistent with the recent result of O'Sullivan \& Green (1999) which gives $-0.6 \% \mathrm{yr}^{-1}$ at $v=15 \mathrm{GHz}$. We therefore conclude that the observed relatively large secular decrease of the flux from Cas A is consistent with the assumption (Shklovsky 1968) that the flux is dominated by the contribution of electrons, produced in a previous epoch, which at the current stage undergo adiabatic cooling. Together with the decrease of the downstream magnetic field this cooling causes the relatively large rate of decrease of the radio flux.

The picture described is reproduced in our model (Fig. 4). One can see that the calculated secular decrease of the flux at $v \lesssim 10 \mathrm{GHz}$ is about $-0.5 \% \mathrm{yr}^{-1}$ and thus very close to the observations. At larger frequencies $v \gtrsim 10^{3} \mathrm{GHz}$ the theoretically calculated decrease has a rather complicated dependence upon $v$ and is on average larger than at low frequencies. The highest secular decline predicted at the largest frequencies $v \gtrsim 10^{16} \mathrm{~Hz}$ is due to synchrotron losses which lead to the decrease of the electron maximum energy.

Since the shock currently propagates through the free red supergiant wind, the contribution of shell electrons to the total synchrotron flux will decrease in time compared with that of the electrons produced by the shock in the free wind. Therefore the rate of secular decline of the total synchrotron flux is expected to decrease from the current value $-0.5 \% \mathrm{yr}^{-1}$ towards $-0.1 \% \mathrm{yr}^{-1}$. 


\subsection{The spatial structure of Cas $A$}

The downstream spatial distribution of radio emitting electrons is qualitatively consistent with the observed structure of Cas A. The brightest part of the remnant in our model is the swept-up shell. It is currently in the downstream region near the contact discontinuity $R_{\mathrm{p}}$ which separates ejecta and swept-up matter. We believe that it corresponds to the observed bright radio ring. The "diffuse plateau" then corresponds to emission coming from the swept-up free wind matter further out.

The small scale irregularities of the radio emission, the socalled compact radio knots (e.g. Andersen \& Rudnick 1996) discussed earlier, which can not be reproduced in our spherically symmetric model, could well be due to amplification of the magnetic field at the bow shocks driven ahead of the socalled fast moving knots, in the form of dense clumps of SNR ejecta. Electrons with energies $\epsilon_{\mathrm{e}}<1 \mathrm{GeV}$, which produce the radio emission, are strongly connected with the moving gas even on such small scales because of their small diffusion coefficient. Therefore their concentration also increases at the bow shock like the gas density. Together with the field amplification this leads to a strong increase of the radio emission from these relatively small volumes.

We note that the field amplification leads to a decreasing effective energy $\epsilon_{\mathrm{e}} \propto \sqrt{v / B}$ of the electrons which produce the synchrotron emission at a given frequency $v$. Together with the adiabatic electron heating, this leads to a brightness increase, and in parallel to a steepening of the radio spectrum because of the concave shape of the electron spectrum in the corresponding energy range. Such a type of correlation between the knot brightness and synchrotron spectral index is indeed observed (Andersen \& Rudnick 1996).

The effect is expected to be larger in the outer downstream region occupied by the swept-up free wind matter and recently accelerated electrons compared with the swept-up shell region, where electrons after their production have already adiabatically cooled by expansion of the medium. Therefore a steeper synchrotron spectrum is expected from the knots situated in the outer diffuse radio plateau surrounding the bright ring, than from the knots in the ring. This is also indicated by the observations (Andersen \& Rudnick 1996).

\subsection{High energy $\gamma$-ray emission from Cas $A$}

Figure 5 represents the expected integral $\gamma$-ray energy flux components from NB, IC scattering on the background radiation field (cosmic microwave + optical/infrared), and hadronic collisions of CR protons with gas nuclei, respectively.

As already mentioned, in contrast with our sphericallysymmetric model one has to expect that not every part of the shock surface efficiently injects and accelerates CRs. At the local portions of the shock, which are currently quasiperpendicular the suprathermal particle injection is presumably suppressed. Therefore the number of accelerated protons calculated within the spherical approach should be corrected by some renormalization factor $f_{\text {re }}<1$. It was argued earlier (Berezhko et al. 2002) that values $f_{\text {re }}=0.15$ to 0.25 are consistent with theoretical considerations (Völk et al., in preparation),

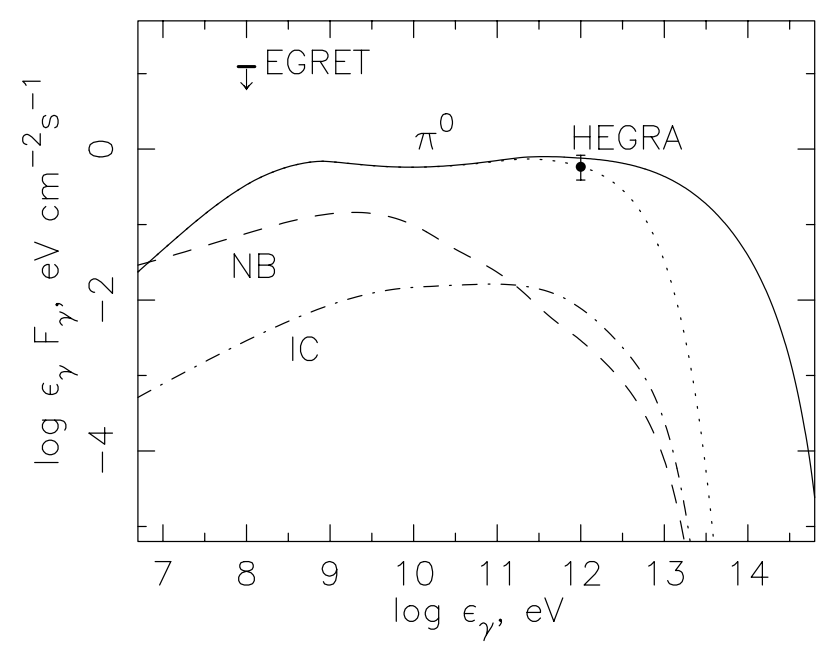

Fig. 5. Inverse Compton (IC, dash-dotted), nonthermal Bremsstrahlung (NB, dashed), and $\pi^{0}$-decay (solid) integral $\gamma$-ray energy fluxes as a function of $\gamma$-ray energy. The dotted line represents the same $\pi^{0}$-decay $\gamma$-ray flux with an exponential cutoff $\exp \left(-\epsilon_{\gamma} / \epsilon_{\gamma}^{\max }\right)$, where $\epsilon_{\gamma}^{\max }=4 \mathrm{TeV}$. The $1 \mathrm{TeV}$ data point is from HEGRA (Aharonian et al. 2001), the EGRET upper limit for $\epsilon_{\gamma}>100 \mathrm{MeV}$ is from Esposito et al. (1996).

the average requirements of the Galactic CR energy budget, and the observed structure of the remnant SN 1006. One of these arguments is that for a typical SNR our spherically symmetric model predicts a significantly larger CR production $E_{\mathrm{c}}=0.3$ to $0.6 E_{\mathrm{sn}}$ than required for the Galactic energy budget $E_{\mathrm{c}} \approx 0.1 E_{\mathrm{sn}}$ (e.g. Berezhko et al. 1996; Berezhko \& Völk 1997, 2000b). The $\pi^{0}$-decay $\gamma$-ray flux presented in Fig. 5 was calculated with a renormalization factor $f_{\text {re }}=1 / 6$. Note that the number of accelerated electrons, which is required for the observed synchrotron flux, remains the same. Therefore the actual electron to proton ratio amounts to $K_{\text {ep }}=0.02$ instead of $4 \times 10^{-3}$. This also means that CRs absorb only $E_{\mathrm{c}}=10^{49} \mathrm{erg}$ at the current epoch.

As one can see from Fig. 5, at $\epsilon_{\gamma}=1 \mathrm{TeV}$ the $\pi^{0}$-decay energy flux has a value of $\epsilon_{\gamma} F_{\gamma} \sim 1 \mathrm{eV} \mathrm{cm}^{-2} \mathrm{~s}^{-1}$, which is close to the experimental value of the reported integral $\mathrm{TeV} \gamma$-ray flux (Aharonian et al. 2001). Also the slope of the $\pi^{0}$-spectrum lies in the reported range of allowed energy spectra, which is quite large due to statistical uncertainties. The $\pi^{0}$-decay energy flux hardens slightly above several $\mathrm{GeV}$; nevertheless its value $\epsilon_{\gamma} F_{\gamma}$ remains practically constant between $300 \mathrm{MeV}$ and several TeV, and has a cutoff energy of about $30 \mathrm{TeV}$.

On the other hand, the IC and NB fluxes at TeV energies are only at levels of $\epsilon_{\gamma} F_{\gamma} \sim 10^{-2} \mathrm{eV} \mathrm{cm}^{-2} \mathrm{~s}^{-1}$ and below. Note that the electron scattering off the infrared/optical background contributes about $50 \%$ to the IC $\gamma$-ray flux at energies $\epsilon_{\gamma}<1 \mathrm{TeV}$. This is by a factor of two larger than the estimate of Gaisser et al. (1998), due to the fact that our electron spectrum is essentially steeper; for such a spectrum, the contribution of background photons with higher energies becomes more important.

The cutoff energy of the IC spectrum is about $2 \mathrm{TeV}$. At $30 \mathrm{TeV}$, the leptonic $\gamma$-ray emission is entirely negligible. The contribution from the CMB target field drops because of the cutoff of the electron spectrum; the scattering off the IR/optical 
photons is suppressed as a result of the decline of the KleinNishina cross section. The cutoff energy of the IC spectrum resulting from the CMB photons is a bit lower than in the case of IR/optical radiation. Therefore at $\epsilon_{\gamma}>1 \mathrm{TeV}$ the contribution of IR/optical background becomes progressively larger compared to the $\mathrm{CMB}$ photons, so that at $\epsilon_{\gamma}=10 \mathrm{TeV}$ it exceeds the CMB contribution by a factor of 200 .

Figure 5 also shows the integral upper limit above $100 \mathrm{MeV}$ as reported by EGRET (Esposito et al. 1996). This limit is well above the $\pi^{0}$-spectrum; even an energy-resolved analysis of the EGRET data - which is not available in the literature yet is not expected to yield upper limits which would violate the model prediction.

Pion production by the accelerated nuclei leads not only to $\gamma$-rays but also to secondary electrons and positrons. However, even for the large gas density $N_{\mathrm{g}} \lesssim 50 \mathrm{~cm}^{-3}$ in the shocked shell, it can be easily shown that the ratio of secondary electrons to protons is more than two orders of magnitude lower than the value $K_{\mathrm{ep}}=0.02$ for primaries deduced here. Therefore the contribution of secondaries to the synchrotron and Inverse Compton emission and the nonthermal Bremsstrahlung is negligible.

\subsection{Remaining model uncertainties}

We believe that there is no significant difference between the calculated and measured $\mathrm{TeV} \gamma$-ray flux of Cas A. Nevertheless we have to ask ourselves to which extent our calculated $\gamma$-ray flux is a robust result and which effects could modify it.

This concerns first of all the magnetic field configuration in the shell and in the free wind region. The RSG wind is most probably driven by strong wave activity from the star's outer convection layers, essentially Alfvén waves (Hartmann \& McGregor 1980).

Their wavelengths should be quite large and unrelated to the gyro radius scale of the accelerated particles. The SNR shock propagates through this turbulent wind and injection occurs at the quasi-parallel portions of the magnetic field lines. For large enough wave amplitudes in the wind, the mean field direction is no more relevant for the acceleration in a strong shock that produces in addition its own large-amplitude resonantly scattering waves (Lucek \& Bell 2000). In the shell the situation is even more pronounced because of the compression by the Wolf-Rayet wind from behind and the resulting hydrodynamic instabilities (Garcia-Segura et al. 1996). We therefore believe that it is justified to use the Bohm limit for the diffusion coefficient, renormalizing the flux since the injection at the instantaneously quasi-perpendicular shock positions is reduced. Thereby we neglect the possible systematic effect of the mean field that in principle has a Parker spiral type configuration with the tendency to further reduce the injection rate.

The second physical effect which can play a role in the current evolutionary phase is CR escape during the more recent phase of the SNR evolution. Let us assume that the red supergiant wind speed $V_{\mathrm{w}} \approx 10 \mathrm{~km} \mathrm{~s}^{-1}$ does not exceed the Alfvén speed, and that the field strength in the free red supergiant wind
$B_{\mathrm{w} 2}$ is as low as $10 \mu \mathrm{G}$. In this case the maximum momentum of accelerated protons

$p_{\max } \propto R_{\mathrm{s}} V_{\mathrm{s}} / \kappa(m c)$

is at least 20 times smaller compared with its value in the shell since the minimum CR diffusion coefficient $\kappa \propto 1 / B$ increases by a factor of 20 . In such a situation one should expect that the diffusion coefficient of particles with $p>p_{\max }^{\mathrm{w}}$, where $p_{\max }^{\mathrm{w}}$ is the maximum momentum of CRs accelerated in the red supergiant wind region $r>R_{2}$, exceeds the Bohm limit because the shock becomes unable to accelerate them and they consequently do not produce high level Alfvénic turbulence near the shock. It is like if the shock had become "old", being unable to confine the highest energy particles it accelerated previously in the shell. Therefore these particles leave the SNR very soon after the $\mathrm{SN}$ shock reaches the red supergiant wind region, resulting in a much smaller production of $\pi^{0}$-decay $\gamma$-rays with the highest energies compared with the previous cases. For the electron X-ray emission this is a small effect since their main synchrotron radiation comes from the shell; their IC emission remains essentially the same with or without escape.

To illustrate this scenario we present in Fig. 5 a $\gamma$-ray spectrum which compared to the previous one has an additional cutoff factor $\exp \left(-\epsilon_{\gamma} / \epsilon_{\gamma}^{\max }\right)$; the cutoff energy $\epsilon_{\gamma}^{\max }$ was set to $4 \mathrm{TeV}$ which corresponds to the escape of particles with energies above $50 \mathrm{TeV}$, lowering the proton maximum energy by a factor of 20. As one can see from Fig. 5 this would still be consistent with the HEGRA flux, but steepen the spectrum at $1 \mathrm{TeV}$.

The integral NB and IC energy fluxes are almost 2 orders of magnitude lower than the corresponding $\pi^{0}$-decay flux. Even taking into account the local thermal far infrared fluxes can not increase the IC flux by more than a factor of 2 (R. J. Tuffs, private communication). Therefore the dominance of the $\pi^{0}$ decay flux is a rather robust result.

\section{Summary}

Perhaps the most important result of our considerations is that the spectral shape of shock accelerated electrons with their essential synchrotron cooling in the downstream region is very well consistent with the observed synchrotron emission. To reproduce a very steep radio spectrum $S_{v} \propto v^{-0.77}$ the shock must be strongly modified. This shock modification can only be produced by accelerated protons if they are also efficiently injected into the acceleration process, as it was assumed in the calculation.

The significant synchrotron losses of electrons in the strong interior magnetic field makes their spectrum steep $N_{\mathrm{e}} \propto \epsilon_{\mathrm{e}}^{-3}$ also at high energies $\epsilon_{\mathrm{e}}>10 \mathrm{GeV}$. This leads to a flat connection of the spectral energy distributions of the observed radio and X-ray synchrotron emissions.

The rather high secular decline of the synchrotron radiation observed in the radio range is naturally reproduced in our model, because this range is dominated by electrons which were accelerated at a previous epoch and undergo currently adiabatic and synchrotron cooling in the expanding downstream region. 
We find that after reduction of the predictions of the nonlinear spherically-symmetric model by a renormalization of the number of accelerated nuclear CRs, to take account of the large areas of quasiperpendicular shock regions of a SNR, good consistency with all observational data can be achieved, including the reported $\mathrm{TeV} \gamma$-ray flux (Aharonian et al. 2001). The used renormalisation factor is consistent with the need that the average acceleration efficiency of a typical SNR within our model scenario should meet the requirements for Galactic CR acceleration.

In addition, our calculations show that at all energies above $1 \mathrm{GeV}$ the $\gamma$-ray production is dominated by $\pi^{0}$-decay. At $\mathrm{TeV}$ energies the expected $\pi^{0}$-decay flux exceeds the IC and NB fluxes by a factor of about seventy. Therefore the leptonic emission is totally inadequate to explain the observed $\mathrm{TeV} \gamma$-ray flux. The $\pi^{0}$-decay spectrum $F_{\gamma}^{\pi} \propto \epsilon_{\gamma}^{-1}$ extends up to $30 \mathrm{TeV}$, whereas the IC and NB $\gamma$-ray fluxes have a cutoff at about $1 \mathrm{TeV}$. Therefore the detection of $\gamma$-ray emission at $10 \mathrm{TeV}$ and above would imply further evidence for its hadronic origin.

We conclude that the observed properties of the radio and $\mathrm{X}$-ray emission can be explained within the assumption that the SN blast wave is the main source of energetic particles in Cas A. The CR production efficiency and the electron to proton ratio implied by these multi-wavelength observations are consistent with the requirements of the nuclear CR sources in the Galaxy.

Acknowledgements. This work has been supported in part by the Russian Foundation for Basic Research (grants 00-02-17728, 9902-16325) and by the Russian Federal Program "Astronomiya" (grant 1.2.3.6). EGB acknowledges the hospitality of the Max-PlanckInstitut für Kernphysik where part of this work was carried out. The authors thank F. Aharonian, W. Hofmann and R. Tuffs for valuable discussions.

\section{References}

Aharonian, F. A., Akhperjanian, A., Barrio, J., et al. 2001, A\&A, 370, 112

Aharonian, F. A., \& Atoyan, A. M. 1999, A\&A, 351, 330

Allen, G. E., Keohane, J. W., Gotthelf, E. V., et al. 1997, ApJ, 487, L97

Andersen, M. C., \& Rudnick, L. 1995, ApJ, 441, 307

Andersen, M. C., \& Rudnick, L. 1996, ApJ, 456, 234

Andersen, M. C., Rudnick, L., Leppik, P., Perley, R. A., \& Braun, R. 1991, ApJ, 373, 146

Atoyan, A. M., Aharonian, F. A., Tuffs, R. J., \& Völk, H. J. 2000a, A\&A, 355, 211

Atoyan, A. M., Tuffs, R. J., Aharonian, F. A., \& Völk, H. J. 2000b, A\&A, 354, 915

Baars, J. W. M., Genzel, R., Paulini-Toth, I. I. K., \& Witzel, A. 1977, A\&A, 61, 99

Bell, A. R., Gull, S. F., \& Kenderline, S. 1975, Nature, 257, 463

Bell, A. R., \& Lucek, S. G. 2001, MNRAS, 321, 433

Bennet, L., \& Ellison, D. C. 1995, JGR, 100, 3439

Berezhko, E. G. 1996, Astropart. Phys., 5, 367

Berezhko, E. G., Elshin, V. K., \& Ksenofontov, L. T. 1996, JETP, 82, 1

Berezhko, E. G., \& Ksenofontov, L. T. 1999, JETPh, 89, 391
Berezhko, E. G., Ksenofontov, L. T., \& Petukhov, S. I. 1999, in Proc. of the 26th ICRC, ed. D. Kieda, M. Salamon, \& B. Dingus (Salt Lake City), 4, 431

Berezhko, E. G., Ksenofontov, L. T., \& Völk, H. J. 2002, A\&A, 395, 943

Berezhko, E. G., Pühlhofer, G., \& Völk, H. J. 2001, in Proc. of the 27th ICRC, Hamburg, 2, 2473

Berezhko, E. G., \& Völk, H. J. 1997, Astropart. Phys., 7, 183

Berezhko, E. G., \& Völk, H. J. 2000a, Astropart. Phys., 14, 201

Berezhko, E. G., \& Völk, H. J. 2000b, A\&A, 357, 283

Berezinskii, V. S., Bulanov, S. A., Dogel, V. A., et al. (eds.) 1990, Astrophysics of cosmic rays (North-Holland: Publ. Comp)

Borkowski, K. J., Szymkowiak, A. E., Blondin, J. M., \& Sarazin, C. L. 1996, ApJ, 466, 866

Braun, R. 1987, A\&A, 171, 233

Butt, Y. M., Torres, D. F., Romero, G. E., et al. 2002, Nature, 418, 499

Chevalier, R. A., \& Kirshner, R. P. 1978, ApJ, 219, 931

Chevalier, R. A., \& Liang, E. P. 1989, ApJ, 344, 332

Cowsik, R., \& Sarkar, S. 1984, MNRAS, 207, 745

Dermer, C. D. 1986, A\&A, 157, 223

Dickel, J. R., \& Greisen, E. W. 1979, A\&A, 75, 44

Drury, L. O., Aharonian, F. A., \& Völk, H. J. 1994, A\&A, 287, 959

Ellison, D. C., Baring, M. G., \& Jones, F. C. 1995, ApJ, 453, 873

Enomoto, R., Tanimori, T., Naito, T., et al. 2002, Nature, 416, 823

Esposito, J. A., Hunter, S. D., Kanbach, G., \& Sreekumar, P. 1996, ApJ, 461, 820

Favata, F., Vink, J., Fiume, D. D., et al. 1997, A\&A, 324, L49

Gaisser, T. K., Protheroe, R. J., \& Stanev, T. 1998, ApJ, 492, 219

Garcia-Segura, G., Langer, N., \& Low, M.-M. M. 1996, A\&A, 316, 133

Hartmann, L., \& McGregor, K. B. 1980, ApJ, 242, 260

Jones, E. M., Smith, B. W., \& Straka, W. C. 1981, ApJ, 249, 185

Jones, T. W., Kang, H., \& Tregillis, I. L. 1994, ApJ, 432, 194

Kassim, N. E., Perley, R. A., Dwarakanath, K. S., \& Erickson, W. C. 1995, ApJ, 455, L59

Kirshner, R. P., \& Chevalier, R. A. 1977, ApJ, 218, 142

Lucek, S. G., \& Bell, A. R. 2000, MNRAS, 314, 65

Malkov, M. A. 1998, Phys. Rev. E, 58, 4911

Malkov, M. A., \& Drury, L. O. 2001, Rep. Prog. Phys., 64, 429

Malkov, M. A., \& Völk, H. J. 1995, A\&A, 300, 605

Malkov, M. A., \& Völk, H. J. 1996, Adv. Space Res., 21(4), 551

Mezger, P. G., Tuffs, R. J., Chini, R., Kreysa, E., \& Gemuend, H.-P. 1986, A\&A, 167, 145

Naito, T., \& Takahara, F. 1994, J. Phys. G: Nucl. Part. Phys., 20, 477

O'Sullivan, C., \& Green, D. A. 1999, MNRAS, 303, 575

Peimbert, M. 1971, ApJ, 170, 261

Peimbert, M., \& van den Bergh, S. 1971, ApJ, 167, 223

Reed, J. E., Hester, J. J., Fabian, A. C., \& Winkler, P. F. 1995, ApJ, 440, 706

Rees, N. 1990, MNRAS, 243, 637

Reimer, O., \& Pohl, M. 2002, A\&A, 390, L43

Scholer, M., Trattner, K. J., \& Kucharek, H. 1992, ApJ, 395, 675

Scott, J. S., \& Chevalier, R. A. 1975, ApJ, 197, L5

Shklovsky, I. S. (ed.) 1968, Supernovae (John Wiley \& Sons Ltd)

Trattner, K. J., \& Scholer, M. 1994, JGR, 99, 6637

Tuffs, R. J. 1986, MNRAS, 219, 13

Tuffs, R. J., Drury, L., Fishera, J., et al. 1997, in Proc. 1st ISO Workshop on Analytical Spectroscopy, ESA SP-419, 177

Vink, J. 1999, Ph.D. Thesis, Leiden Univ.

Vink, J., Kaastra, J. S., \& Bleeker, J. A. M. 1996, A\&A, 307, L41

Völk, H. J., Berezhko, E. G., Ksenofontov, L. T., \& Rowell, G. P. 2002, A\&A, 396, 649 